\title{
DC and Hall conductivity in holographic massive Einstein-Maxwell-Dilaton gravity
}

\author{
Zhenhua Zhou, ${ }^{a}$ Jian-Pin $\mathbf{W} \mathbf{u}^{b, c}$ and Yi Ling ${ }^{a, c}$ \\ ${ }^{a}$ Institute of High Energy Physics, Chinese Academy of Sciences, \\ Beijing 100049, China \\ ${ }^{b}$ Institute of Gravitation and Cosmology, Department of Physics, \\ School of Mathematics and Physics, \\ Bohai University, Jinzhou 121013, China \\ ${ }^{c}$ State Key Laboratory of Theoretical Physics, Institute of Theoretical Physics, \\ Chinese Academy of Sciences, Beijing 100190, China \\ E-mail: zhouzh@ihep.ac.cn, jianpinwu@mail.bnu.edu.cn, lingy@ihep.ac.cn
}

ABSTRACT: We investigate the holographic DC and Hall conductivity in massive EinsteinMaxwell-Dilaton (EMD) gravity. Two special EMD backgrounds are considered explicitly. One is dyonic Reissner-Nordström-AdS (RN-AdS) geometry and the other one is hyperscaling violation AdS (HV-AdS) geometry. We find that the linear-T resistivity and quadraticT inverse Hall angle can be simultaneously achieved in HV-AdS models, providing a hint to construct holographic models confronting with the experimental data of strange metal in future.

KEYwORDS: Holography and condensed matter physics (AdS/CMT), AdS-CFT Correspondence

ArXiv ePrint: 1504.00535 


\section{Contents}

1 Introduction 1

2 DC and Hall conductivity in holographic massive EMD gravity 2

3 DC and Hall conductivity in some specific holographic models $\quad 5$

3.1 DC and Hall conductivity in dyonic RN-AdS geometry 5

3.2 DC and Hall conductivity in dyonic HV-AdS geometry 6

4 Conclusions and discussions $\quad 8$

A The dyonic HV-AdS solution of massive EMD gravity 9

\section{Introduction}

The strange metal phase, which emerges in normal states of high temperature superconductors and heavy fermion compounds near a quantum critical point, exhibits a number of weird transport properties that challenge understanding. The most famous characteristics of the strange metal phase is that its resistivity $\rho(T)$ varies linearly with temperature, while the inverse of Hall angle $\theta_{H}^{-1}(T)$ varies quadratically with temperature [1-4]. Some theoretical attempts have been made to attack this problem by proposing that there are different scalings between Hall angle and resistivity [5, 6]. However, due to the strongly correlated nature in these materials, a complete resolution to this problem is still absent in theory so far.

AdS/CFT correspondence provides a powerful tool to study strongly correlated systems. In particular, the methods of computing transport coefficients by holography have been developed in $[7,8]$. The linear-T resistivity has been solely reproduced by holography in $[9,10]$. One mechanism is to consider a Lifshitz gravitational system with Dirac-BornInfeld (DBI) action, in which the resistivity displays linear property with temperature when the Lifshitz exponent takes a special value of $z=2$ [9]. An alternative is proposed in [10]. They introduced a hydrodynamic state with a minimal viscosity $\eta \sim s$ being weakly coupled to disorder, in which the viscosity will contribute to the resistivity such that $\rho \sim \eta \sim s$. And then by holography, a well-controlled locally quantum critical state with $s \sim T$ [11] is introduced such that one has $\rho \sim T$, i.e., the linear- $T$ resistivity. More recently, progress has been made in addressing different scalings between Hall angle and resistivity by holography [12-23]. Particularly, a scaling analysis suggests that the anomalous behaviors $\rho \sim T$ as well as $\theta_{H} \sim 1 / T^{2}$ can be reproduced in the HV geometry with DBI action [21]. But note that the backreaction of gauge field on the spacetime geometry is ignored in [21]. Another progress is from ref. [23], in which the resistivity and Hall angle exhibit different scalings in holographic Q-lattice model. 
Inspired by the work in [23], in present paper we attempt to address this dichotomy between resistivity and the Hall angle in a simpler holographic framework, i.e., massive gravity. The first holographic massive gravity model is constructed in [24] where a finite DC conductivity is observed. Subsequently, a lot of works have also implemented finite DC conductivity in this framework [25-31]. Due to the breaking of diffeomorphism symmetry, the stress-energy tensor in massive gravity is not conserved, leading to a similar effect of dissipating momentum as that of holographic lattice model [18, 32-43], which has originally been pointed out in [44]. Here, we shall first derive general analytic expressions for the resistivity and the Hall angle in holographic massive EMD gravity in section 2. These expressions are applicable for a larger class of scaling geometries. Specifically, we shall discuss the case of dyonic RN-AdS and HV-AdS geometry in section 3. We conclude this paper with a brief discussion on the scale dimension analysis at low temperature.

Note added: while this work was in preparation ref. [45] appeared, which has some overlap with ours. In addition, we would like to point out that the thermoelectric conductivities at finite magnetic field in holographic Q-lattice model were discussed in [46, 47].

\section{DC and Hall conductivity in holographic massive EMD gravity}

In this section, we shall derive general analytic expressions for DC and Hall conductivity in holographic massive gravity, which can be applicable for a large class of scaling geometries. For this purpose, we generalize the holographic massive gravity action in [24] to the following EMD theory,

$$
S=\frac{1}{2 \kappa^{2}} \int d^{4} x \sqrt{-g}\left[R-\frac{Z(\phi)}{4} F^{2}+\frac{1}{2}(\partial \phi)^{2}+V(\phi)+\beta(\phi)\left([\mathcal{K}]^{2}-\left[\mathcal{K}^{2}\right]\right)\right] .
$$

where $[\mathcal{K}]:=\mathcal{K}^{\mu}{ }_{\mu}$ and $\left[\mathcal{K}^{2}\right]:=\left(\mathcal{K}^{2}\right)^{\mu}{ }_{\mu}$ with $\mathcal{K}^{\mu}{ }_{\nu}:=\sqrt{g^{-1} f}{ }_{\nu}{ }_{\nu}$ and $\left(\mathcal{K}^{2}\right)_{\mu \nu}:=\mathcal{K}_{\mu \alpha} \mathcal{K}^{\alpha}{ }_{\nu} . f_{\mu \nu}$ is the reference metric and we are interested in the special case with $f_{\mu \nu}=\operatorname{diag}(0,0,1,1)$, which breaks the diffeomorphism along two spatial directions. In comparison with [24, 45], a scalar field is introduced with an arbitrary potential $V(\phi)$, a dilaton-like coupling $Z(\phi)$ as well as a dilaton dependent coupling parameter $\beta(\phi)$ of massive term, which is necessary to obtain a dyonic HV-AdS black hole solution in our setup. When $\beta$ is a constant, the bulk theory is ghost-free because the lapse function can be a Lagrange multiplier by choosing a special shift vector, which is demonstrated in [24]. For details, we refer to ref. [24] and references therein. When $\beta(\phi)$ is a function of scalar field $\phi$, we also find that our theory (2.1) is ghost-free since $\beta(\phi)$ is still independent of the lapse function.

To study the Hall conductivity, we turn on a magnetic field on the background and consider the following ansatz

$$
\begin{aligned}
d s^{2} & =-U(r) d t^{2}+V(r) d r^{2}+W(r)\left(d x^{2}+d y^{2}\right), \\
A & =a(r) d t+B x d y, \quad \phi=\phi(r),
\end{aligned}
$$

where $B$ is a constant magnetic field. We also assume that $U, V, W>0$ and at the horizon position $r_{+}, U\left(r_{+}\right)=1 / V\left(r_{+}\right)=0, U\left(r_{+}\right) V\left(r_{+}\right)<\infty$. These assumptions are usually true for general holographic models. 
In order to compute the DC and Hall conductivity, we consider vector fluctuations over the homogeneous and isotropic background (2.2) and (2.3). Due to the presence of magnetic field, these perturbations will induce electric currents along $x$ and $y$ directions and provide non-zero contributions to the $t-x, t-y, r-x, r-y$ components of the energy-momentum tensor. It turns out that a consistent ansatz for perturbations can be chosen as

$$
\begin{aligned}
A_{x} & =-E_{x} t+a_{x}(r), & A_{y} & =a_{y}(r), \\
\delta g_{t x} & :=W h_{t x}(r), & \delta g_{r x} & :=W h_{r x}(r), \\
\delta g_{t x} & :=W h_{t y}(r), & \delta g_{r y} & :=W h_{r y}(r) .
\end{aligned}
$$

Here, following closely the method outlined in [23, 39], we turn on a constant electric field $E_{x}$ to detect the DC and Hall conductivity.

With the use of Maxwell equations, one can define the conserved charge $Q$ and the conserved currents $J_{x}, J_{y}$ as follows

$$
\begin{aligned}
Q & :=-Z(\phi) \frac{W}{\sqrt{U V}} a^{\prime}, \\
J_{x} & :=Q h_{t x}-Z(\phi) B \sqrt{\frac{U}{V}} h_{r y}-Z(\phi) \sqrt{\frac{U}{V}} a_{x}^{\prime}, \\
J_{y} & :=Q h_{t y}+Z(\phi) B \sqrt{\frac{U}{V}} h_{r x}-Z(\phi) \sqrt{\frac{U}{V}} a_{y}^{\prime},
\end{aligned}
$$

where the prime denotes the derivative with respect to $r$. The conductivities along $x$ and $y$ directions can be expressed as $\sigma_{x x}=J_{x} / E_{x}, \sigma_{x y}=J_{y} / E_{x}$, respectively. Because $J_{x}, J_{y}$ are conserved along $r$ direction, it is more convenient to evaluate them at $r=r_{+}$. Thus, the conductivities can be completely determined by the regularity of fluctuation modes at the horizon, which are

$$
\begin{aligned}
a_{x}^{\prime} & =-\sqrt{\frac{U}{V}} E_{x}+\mathcal{O}\left(r_{+}-r\right), & a_{y}^{\prime} & =\mathcal{O}\left(r_{+}-r\right), \\
h_{r x}\left(r_{+}\right) & =\sqrt{\frac{U}{V}} h_{t x}\left(r_{+}\right), & h_{r y}\left(r_{+}\right) & =\sqrt{\frac{U}{V}} h_{t y}\left(r_{+}\right) .
\end{aligned}
$$

The currents now can be expressed as

$$
\begin{aligned}
& J_{x}=Q h_{t x}\left(r_{+}\right)-\left.Z(\phi)\right|_{r_{+}} B h_{t y}\left(r_{+}\right)+\left.Z(\phi)\right|_{r_{+}} E_{x}, \\
& J_{y}=Q h_{t y}\left(r_{+}\right)+\left.Z(\phi)\right|_{r_{+}} B h_{t x}\left(r_{+}\right) .
\end{aligned}
$$

Taking into account the $x-x, r-x, r-y$ components of Einstein equation and evaluating them at $r=r_{+}$, we can derive the relations between the electric perturbation and metric perturbation as,

$$
\begin{aligned}
\left(B^{2} Z+m\right) h_{t x}+Q B h_{t y} & =Q E_{x} \\
Q B h_{t x}-\left(B^{2} Z(\phi)+m\right) h_{t y} & =-Z B E_{x}
\end{aligned}
$$


where all the variables should be understood as taking values at $r=r_{+}$and the variable $m$ is defined as $m:=-2 \beta(\phi) W(r)$. Putting these solutions into (2.12) and (2.13), we obtain the conductivity as

$$
\begin{aligned}
\sigma_{x x} & =\left.\frac{m\left(B^{2} Z^{2}+Q^{2}+Z m\right)}{\left(B^{2} Z+m\right)^{2}+B^{2} Q^{2}}\right|_{r_{+}}, \\
\sigma_{x y} & =\left.\frac{B Q\left(B^{2} Z^{2}+Q^{2}+2 Z m\right)}{\left(B^{2} Z+m\right)^{2}+B^{2} Q^{2}}\right|_{r_{+}} .
\end{aligned}
$$

Finally, the Hall angle $\theta_{H}:=\sigma_{x x} / \sigma_{x y}$ and DC conductivity $\sigma_{D C}:=\sigma_{x x}(B=0)$ are derived as

$$
\begin{aligned}
\theta_{H} & =\left.\left.\frac{B Q\left(B^{2} Z^{2}+Q^{2}+2 Z m\right)}{m\left(B^{2} Z^{2}+Q^{2}+Z m\right)}\right|_{r_{+}} \simeq \frac{B Q}{m}\right|_{r_{+}}, \\
\sigma_{D C} & =\left.Z(\phi)\right|_{r_{+}}+\left.\frac{Q^{2}}{m}\right|_{r_{+}} .
\end{aligned}
$$

A similar result has been reported in [23], in which a Q-lattice is introduced instead of massive term to dissipate the momentum. In fact, it has been shown by Andrade and Withers in [37] that the conductivity in massive gravity is equivalent to that of a linear axion model, which could be viewed as a special case of Q-lattices.

Furthermore, we can express the DC conductivity $\sigma_{D C}$ in terms of the thermodynamical quantities and the generic physical parameters as

$$
\sigma_{D C}=\sigma_{c c s}+\frac{Q^{2}}{\mathcal{E}+\mathcal{P}} \tau_{L}
$$

where $s=4 \pi W\left(r_{+}\right), \mathcal{E}$ and $\mathcal{P}$ are the entropy density, energy density and pressure of the system, respectively. In above equation, we have defined a lattice timescale $\tau_{L},{ }^{1}$

$$
\tau_{L}^{-1}=-\frac{s \beta}{2 \pi(\mathcal{E}+\mathcal{P})} .
$$

It is interesting enough to notice that the coefficient function $\beta(\phi)$ in massive term plays the same role as the lattice parameter [23], which has also been revealed in [44]. Also, we defined

$$
\sigma_{c c s}=\left.Z(\phi)\right|_{r_{+}}
$$

which is proposed as a charge-conjugation symmetric conductivity in [23] and independent of the lattice timescale $\tau_{L}[51,52]$. Moreover, the Hall angle $\theta_{H}$ can be expressed in terms of the momentum relaxation timescale as

$$
\theta_{H}=\frac{B Q}{\mathcal{E}+\mathcal{P}} \tau_{L}
$$

So far, we have successfully implemented the dichotomy between DC resistivity and the Hall angle in the framework of holographic massive gravity, which provides a new

\footnotetext{
${ }^{1} \tau_{L}$ can be interpreted as a momentum relaxation rate only in the hydrodynamic regime, namely at slow momentum dissipation [50].
} 
mechanism to realize the anomalous transport behaviors of strange metal. Since the functions $Z(\phi), \beta(\phi)$ in eqs. (2.18) and (2.19)(or $\sigma_{c c s}, \tau_{L}$ in eqs. (2.20) and (2.23)) are quite general and adjustable, following the ideas proposed in [53-55], we can build an effective holographic model confronting with the experimental data. Specifically, we will investigate the temperature dependence of conductivity and Hall angle over a dyonic RN-AdS black hole and dyonic HV-AdS black hole in next sections. In this circumstance, one needs to fix either the chemical potential or the charge density of the system. From a phenomenological point of view we intend to perform the analysis with a fixed charge density since most of the experimental setup for cuprates are at constant charge density, for instance, as described in [56].

\section{DC and Hall conductivity in some specific holographic models}

In this section, we shall consider two explicit massive EMD models in RN-AdS and HV-AdS background, respectively. The corresponding temperature dependence of DC conductivity and Hall angle are obtained via the general expressions above. Especially, we find that the linear-T resistivity and quadratic- $\mathrm{T}$ inverse Hall angle can be achieved simultaneously in a HV model with $z=6 / 5$ and $\theta=8 / 5$.

\subsection{DC and Hall conductivity in dyonic RN-AdS geometry}

For the asymptotic AdS case, we can choose

$$
d s^{2}=\frac{1}{r^{2}}\left(-f(r) d t^{2}+\frac{d r^{2}}{f(r)}+d x^{2}+d y^{2}\right) .
$$

A background solution for the action with $V(\phi)=6, Z(\phi)=1, \beta(\phi):=\beta=$ const. in eq. (2.1) is

$$
\begin{aligned}
& f(r)=1+\beta r^{2}-M r^{3}+\frac{Q^{2}+B^{2}}{4} r^{4}, \\
& a(r)=\mu-Q r
\end{aligned}
$$

where the integral constants $\mu, Q$ can be interpreted as the chemical potential and charge density respectively, while the mass $M$ is determined by $f\left(r_{+}\right)=0$. The Hawking temperature is

$$
T=\frac{3}{4 \pi r_{+}}\left(1+\frac{\beta}{3} r_{+}^{2}-\frac{Q^{2}+B^{2}}{12} r_{+}^{4}\right)
$$

A straightforward usage of expressions (2.18) and (2.19) leads to

$$
\begin{aligned}
\theta_{H} & \simeq-\frac{B Q}{2 \beta} r_{+}^{2}, \\
\sigma_{D C} & =1-\frac{Q^{2}}{2 \beta} r_{+}^{2} .
\end{aligned}
$$


We are interested in high temperature region, in which we have the relation $r_{+} \sim 1 / T$. Therefore, when momentum relaxation is weak (i.e $\sigma_{D C} \simeq-\frac{Q^{2}}{2 \beta} r_{+}^{2}$ ), the resistivity and inverse Hall angle would have the similar behavior of temperature dependence. While when momentum relaxation is strong, the conductivity $\sigma_{D C} \simeq 1$ is independent of the temperature. So, for both cases, it is hardly possible to reproduce the linear-T resistivity and quadratic- $\mathrm{T}$ inverse Hall angle simultaneously.

\subsection{DC and Hall conductivity in dyonic HV-AdS geometry}

Now, we turn to consider the DC and Hall conductivity in dyonic HV-AdS geometry including massive term. For this purpose, we choose

$$
d s^{2}=r^{\theta}\left(-\frac{f(r) d t^{2}}{r^{2 z}}+\frac{d r^{2}}{r^{2} f(r)}+r^{-2}\left(d x^{2}+d y^{2}\right)\right)
$$

where $\theta$ and $z$ are the HV exponent and Lifshitz dynamical exponent, respectively. The setup of the electromagnetic field and scalar field is still given by eq. (2.3). In order to obtain a dyonic HV-AdS solution in an analytical manner, we parameterize the coupling functions and the potential in the action as

$$
Z(\phi)=Z_{0} e^{\lambda \phi}, \quad \beta(\phi)=\beta_{0} e^{\sigma \phi}, \quad V(\phi)=V_{1} e^{\gamma_{1} \phi}+V_{2} e^{\gamma_{2} \phi} .
$$

An asymptotic HV-AdS black hole solution exists only when the following relations are satisfied

$$
\begin{aligned}
\lambda & =(\theta-2 z+2) / \alpha, \\
\sigma & =-2 / \alpha, \\
\gamma_{1} & =-\theta / \alpha \\
\gamma_{2} & =(-\theta-2 z+6) / \alpha, \\
\beta_{0} & =(z-1)(\theta-z-2), \\
V_{1} & =(\theta-2 z)(\theta-z-2), \\
V_{2} & =\frac{\theta-2 z+2}{4(-z+2)} B^{2} Z_{0} .
\end{aligned}
$$

Therefore, the HV model considered here is the action in eq. (2.1) with the parameters satisfying relations (3.9)-(3.15). Furthermore, we have the following analytic dyonical HV-AdS black hole solution ${ }^{2}$

$$
\begin{aligned}
e^{\phi} & =r^{\alpha}, \quad \alpha:=\sqrt{(2-\theta)(\theta-2 z+2)}, \\
a(r) & =\mu-\frac{Q}{Z_{0}(z-\theta)} r^{z-\theta}, \\
f(r) & =1-M r^{-\theta+z+2}+\frac{Q^{2} r^{-2 \theta+2 z+2}}{2 Z_{0}(\theta-2)(\theta-z)}+\frac{B^{2} Z_{0} r^{-2 z+6}}{4(2-z)(\theta-3 z+4)},
\end{aligned}
$$

\footnotetext{
${ }^{2}$ A detailed derivation of the dyonical HV-AdS black hole solution is given in the appendix.
} 
The Hawking temperature is given by

$$
T=\frac{-\theta+z+2}{4 \pi r_{+}^{z}}\left(1+\frac{(z-\theta) Q^{2} r_{+}^{-2 \theta+2 z+2}}{2(\theta-z-2)(\theta-2)(\theta-z)}+\frac{(\theta-3 z+4) B^{2} r_{+}^{-2 z+6}}{4(\theta-z-2)(2-z)(\theta-3 z+4)}\right) .
$$

Before proceeding, we present several remarks on the dyonic HV-AdS black hole solution obtained here. Firstly, we emphasize that for a given bulk action (eq. (2.1)), it is only possible to construct the above dyonic solution (eq. (3.16)-(3.18)) for one particular value of the magnetic field $B$ satisfying eq. (3.15) (but at generic $T$ and $Q$ ). Secondly, we calculate the specific heat of this black hole and find that only for small $B$ as well as $\theta<2$, the specific heat is positive. It indicates that the black hole is thermodynamically stable only for weak magnetic field and $\theta<2$. Thirdly, it is easy to check that this dyonic HV-AdS black hole shares the same near horizon geometry with dyonic RN-AdS black hole, i.e., $A d S_{2} \times \mathbb{R}^{2}$, but the asymptotic geometry (UV) is hyperscaling violating characterized by the Lifshitz exponent $z$ and the HV exponent $\theta$. Note that a different solution for charged HV-AdS black hole with IR being $A d S_{2} \times \mathbb{R}^{2}$ has previously been obtained in [57], and other relevant investigations on EDM models can be found in [58-61]. In addition, from eq. (3.13) one finds that when $z=1$ and $\theta=0$, then $\beta(\phi)=0$ and the model goes back to that without momentum dissipation.

Using eqs. (2.18) and (2.19) in section 2, the Hall angle and DC conductivity for the HV model can be expressed as

$$
\begin{aligned}
\theta_{H} & \simeq \frac{B Q r_{+}^{4-\theta}}{2(z-1)(2+z-\theta)}, \\
\sigma_{D C} & =Z_{0} r_{+}^{\theta-2 z+2}+\frac{Q^{2} r_{+}^{4-\theta}}{2(z-1)(2+z-\theta)} .
\end{aligned}
$$

Obviously, when $z=1$, the DC conductivity becomes infinity due to the absence of momentum dissipation.

Now, we consider the case when the charge-conjugation symmetric conductivity $\sigma_{c c s}$, i.e., the first term in (3.21), is dominant over the second one. Using the relation $r_{+} \sim T^{-1 / z}$ in HV geometry, we have

$$
\theta_{H}^{-1} \sim T^{\frac{4-\theta}{z}}, \quad \rho_{D C}=\sigma_{D C}^{-1} \sim T^{\frac{\theta-2 z+2}{z}} .
$$

Therefore, the linear- $T$ resistivity and quadratic- $T$ inverse Hall angle can be simultaneously achieved by setting $z=6 / 5$ and $\theta=8 / 5$ in our holographic massive EMD model with a specific magnetic field $B$.

Finally, we shall provide a scale analysis to ensure that the results satisfy the scaling law of HV background. Following the method proposed in [21, 22], we introduce the scale dimension of the coordinates and the electric scalar potential as follows,

$$
[x]=-1, \quad[t]=-z, \quad\left[d s^{2}\right]=-\theta, \quad\left[A_{t}\right]=z-\Phi .
$$


The scaling of space and time are assigned in terms of the dispersion relation $\omega \sim k^{z}[9$, $21,22]$. The HV exponent $\theta$ characterizes the scaling of the entropy density. In addition, an anomalous scaling $\Phi$ is also introduced for the uniform scaling transformation of the action. For the detailed discussion, please refer to [21, 22]. From above equations, one can derive the scale dimension of other electric variables as

$$
\begin{array}{ll}
{[E]=1+z-\Phi,} & {[B]=2-\Phi} \\
{[Q]=2-\theta+\Phi,} & {[J]=z-\theta+\Phi+1 .}
\end{array}
$$

Then, by $\sigma=J / E$, the scale dimension of the conductivity is

$$
[\sigma]=-\theta+2 \Phi
$$

The Hall angle is dimensionless because it is the ratio of two conductivities $\sigma_{x x}, \sigma_{x y}$. Note that our results are consistent with those in [21, 22] with $d=2$.

Now, we check the scale behavior of the DC conductivity (3.21) and the Hall angle (3.20). It is easy to check the second term in eq. (3.21) has the same scale dimension as that of the conductivity (3.26). For the first term in eq. (3.21), to have the same scale dimension as that of the conductivity (3.26), we require

$$
[Z(\phi)]=-\theta+2 \Phi \quad \text { or } \quad\left[Z_{0}\right]=-2 z+2+2 \Phi,
$$

which is consistent with the uniform scaling transformation condition

$$
\theta=[R]=\left[(\partial \phi)^{2}\right]=\left[\beta(\phi) r^{-\theta+2}\right]=[V(\phi)]=\left[Z(\phi) F^{2}\right] .
$$

Also, it is direct to check that the Hall angle (3.20) we obtained here is dimensionless.

\section{Conclusions and discussions}

In this paper we have presented a mechanism to implement the dichotomy between the DC resistivity and the Hall angle in massive EMD gravity theory, where the diffeomorphism symmetry is broken along spatial directions and the momentum of the system has dissipation. Following closely the method performed in [39], we have derived general analytic expressions for the DC and Hall conductivity which can be applicable for a large class of holographic massive models. Because both gauge field coupling $Z(\phi)$ and massive coupling $\beta(\phi)$ are completely free and undetermined, this mechanism can provide a viable road toward an effective holographic field theory confronting with the experimental data.

As examples, we have presented a detailed analysis on the scaling behavior of the DC resistivity and Hall angle in dyonic RN-AdS black hole and dyonic HV-AdS black hole, respectively. The reproduction of both linear-T resistivity and quadratic- $\mathrm{T}$ inverse Hall angle in dyonic RN-AdS geometry is still suspensive. However, some surprise occurs in the case of the dyonic HV-AdS geometry including massive gravity term, in which the linear-T resistivity and quadratic-T inverse Hall angle can be simultaneously obtained for $z=6 / 5$ and $\theta=8 / 5$ at large $\sigma_{c c s}$. However, we would like to point out that these scaling behaviors 
hold only in a specific dyonic HV-AdS geometry with one value of $B$. In the future, we expect to search for a general dyonic HV-AdS geometry with arbitrary $B$, in which we can achieve simultaneously these scaling behaviors of strange metal.

We also remark that in this paper a relation $r_{+} \sim 1 / T$ has been applied as in most previous literature $[21,22,62]$. It is a good approximation at high temperature when the field parameters $Q$ and $\beta$ are much smaller than $T$ such that the terms containing $\beta$ and $Q$ in Hawking temperature (3.4) can be ignored. However, at low temperature we must cautiously realize that this relation may not hold anymore. In this circumstance, one could consider the Taylor expansion of thermal observables in powers of the scale invariant quantity such as $T / \sqrt{Q}$ to obtain the behavior of temperature dependence [37].

\section{Acknowledgments}

We are grateful to the anonymous referees for valuable suggestions and comments, which are important in improving our work. We also thank A. Amoretti and A. Karch for helpful discussions. This work is supported by the Natural Science Foundation of China under Grant Nos.11275208, 11305018 and 11178002. Y.L. also acknowledges the support from Jiangxi young scientists (JingGang Star) program and 555 talent project of Jiangxi Province. J. $\mathrm{P}$. Wu is also supported by Program for Liaoning Excellent Talents in University (No. LJQ2014123).

\section{A The dyonic HV-AdS solution of massive EMD gravity}

In this appendix, we present a detailed derivation about the dyonic HV-AdS solution in 3.2. Firstly, we rewrite the action in eq. (2.1) as follows

$$
S=\int d^{4} x \sqrt{-g}\left(R-\frac{Z(\phi)}{4} F^{2}+\frac{1}{2}(\partial \phi)^{2}+V(\phi)+2 \beta(\phi) \sqrt{g^{x x} g^{y y}}\right) .
$$

To obtain a dyonic HV-AdS solution, we parameterize $Z(\phi), V(\phi)$ and $\beta(\phi)$ by exponentials in terms of $\left(\lambda, \sigma, \gamma_{1}, \gamma_{2}, Z_{0}, \beta_{0}, V_{1}, V_{2}\right)$ (see eq. (3.8)). At the same time, we assume a dyonic HV-AdS ansatz

$$
\begin{aligned}
d s^{2} & =r^{\theta}\left(\frac{-f(r)}{r^{2 z}} d t^{2}+\frac{d r^{2}}{f(r) r^{2}}+r^{-2}\left(d x^{2}+d y^{2}\right)\right), \\
A & =a(r) d t+B x d y, \quad \phi=\phi(r)
\end{aligned}
$$

where we have parameterized the metric by the Lifshitz dynamical exponent $z$ as well as the HV exponent $\theta$ and introduced a constant magnetic field $B$.

Applying the variational approach to the action in (A.1), one can derive the Einstein equations as

$$
R_{\nu}^{\mu}=M_{\nu}^{\mu}-S_{\nu}^{\mu}
$$


with

$$
\begin{aligned}
M_{\mu \nu} & \equiv \frac{Z}{2}\left(F_{\mu \alpha} F_{\nu}{ }^{\alpha}-\frac{1}{4} g_{\mu \nu} F^{2}\right), \\
S_{\mu \nu} & \equiv \frac{\delta \mathcal{L}_{m}}{\delta g_{\mu \nu}}-\frac{1}{2} g_{\mu \nu} g^{\alpha \beta} \frac{\delta \mathcal{L}_{m}}{\delta g_{\alpha \beta}}+\frac{1}{2} g_{\mu \nu} \mathcal{L}_{m}, \\
\mathcal{L}_{m} & \equiv \frac{1}{2}(\partial \phi)^{2}+V(\phi)+2 \beta(\phi) \sqrt{g^{x x} g^{y y}} .
\end{aligned}
$$

Using the ansatz (A.2) and (A.3), $M_{\nu}^{\mu}$ and $S^{\mu}{ }_{\nu}$ can be expressed as

$$
\begin{aligned}
M_{\nu}^{\mu} & =\frac{g^{x x} g^{y y}\left(Q^{2}+B^{2} Z^{2}\right)}{4 Z} \operatorname{diag}\{-1,-1,1,1\}, \\
S^{\mu}{ }_{\nu} & =\operatorname{diag}\left\{\frac{V}{2}, \frac{1}{2} g^{r r}\left(\phi^{\prime}\right)^{2}+\frac{V}{2}, \beta \sqrt{g^{x x} g^{y y}}+\frac{V}{2}, \beta \sqrt{g^{x x} g^{y y}}+\frac{V}{2}\right\},
\end{aligned}
$$

where the conserved charge $Q \equiv-Z \sqrt{-g} g^{t t} g^{r r} a^{\prime}$ has been defined using the Maxwell equations. Thus, we can express the Einstein equations as

$$
\begin{aligned}
& R_{t}^{t}=-\frac{g^{x x} g^{y y}\left(Q^{2}+B^{2} Z^{2}\right)}{4 Z}-\frac{V}{2}, \\
& R_{r}^{r}=-\frac{g^{x x} g^{y y}\left(Q^{2}+B^{2} Z^{2}\right)}{4 Z}-\frac{1}{2} g^{r r}\left(\phi^{\prime}\right)^{2}-\frac{V}{2}, \\
& R_{x}^{x}=\frac{g^{x x} g^{y y}\left(Q^{2}+B^{2} Z^{2}\right)}{4 Z}-\beta \sqrt{g^{x x} g^{y y}}-\frac{V}{2}, \\
& R_{y}^{y}=\frac{g^{x x} g^{y y}\left(Q^{2}+B^{2} Z^{2}\right)}{4 Z}-\beta \sqrt{g^{x x} g^{y y}}-\frac{V}{2},
\end{aligned}
$$

as well as the scalar field equation

$$
\triangle \phi=\frac{\dot{Z} g^{x x} g^{y y}\left(Q^{2}-B^{2} Z^{2}\right)}{2 Z^{2}}+2 \dot{\beta} \sqrt{g^{x x} g^{y y}}+\dot{V},
$$

where $\triangle$ is the Laplace operator and dot denotes the derivative with respect to $\phi$. To solve them, we will use the following two important relations

$$
\begin{aligned}
R_{r}^{r}-R_{t}^{t} & =\frac{f}{2 r^{\theta}}(\theta-2)(\theta-2 z+2), \\
r\left(R_{x}^{x}\right)^{\prime}+2(\theta-z) R_{x}^{x} & =(\theta-2) R_{t}^{t} .
\end{aligned}
$$

From the first one and eqs. (A.10) and (A.11), we can obtain the solution of $\phi$

$$
\phi^{\prime}=\alpha / r, \quad \alpha^{2} \equiv(2-\theta)(\theta-2 z+2) .
$$

Since the second relation (A.16) together with eq. (A.12) implies eq. (A.10), we leave only two equations

$$
\begin{aligned}
\frac{2-\theta}{2 r^{\theta}}\left((\theta-z-2) f+r f^{\prime}\right) & =\frac{\left(Q^{2}+B^{2} Z^{2}\right)}{4 Z r^{2(\theta-2)}-\beta r^{2-\theta}-\frac{V}{2}} \\
\frac{r Z^{\prime}\left(Q^{2}-B^{2} Z^{2}\right)}{4 Z^{2} r^{2(\theta-2)}}+\beta^{\prime} r^{3-\theta}+\frac{V^{\prime} r}{2} & =(\theta-2 z+2)\left(\frac{Q^{2}+B^{2} Z^{2}}{4 Z r^{2(\theta-2)}}-\beta r^{2-\theta}-\frac{V}{2}\right),
\end{aligned}
$$


where the first one is from the $\mathrm{x}-\mathrm{x}$ component of the Einstein equation (A.12) and the second one comes from the scalar field equation (A.14).

Now, substituting the solution of $\phi$ (eq. (A.17)) into the coupling functions and the potential, one has

$$
Z(\phi)=Z_{0} r^{\lambda}, \quad \beta(\phi)=\beta_{0} r^{\sigma}, \quad V(\phi)=V_{1} r^{\gamma_{1}}+V_{2} r^{\gamma_{2}},
$$

Then, eq. (A.18) gives

$$
\begin{aligned}
f(r)= & -\frac{Q^{2} r^{4-\theta-\lambda}}{2 Z_{0}(\theta-2)(2-\lambda-z)}-\frac{B^{2} Z_{0} r^{4-\theta+\lambda}}{2(\theta-2)(2+\lambda-z)} \\
& +\frac{2 \beta_{0} r^{2+\sigma}}{(\theta-2)(\theta+\sigma-z)}+\frac{V_{1} r^{\theta+\gamma_{1}}}{(\theta-2)\left(2 \theta+\gamma_{1}-z-2\right)} \\
& +\frac{V_{2} r^{\theta+\gamma_{2}}}{(\theta-2)\left(2 \theta+\gamma_{2}-z-2\right)}-M r^{-\theta+z+2},
\end{aligned}
$$

where $M$ is an integral constant. Eq. (A.19) becomes a polynomial equation of $r$

$$
\begin{array}{r}
(\theta-2 z+2-\lambda) \frac{Q^{2} r^{4-\theta-\lambda}}{2 Z_{0}}+(\theta-2 z+2+\lambda) \frac{B^{2} Z_{0} r^{4-\theta+\lambda}}{2}-(\theta-2 z+2+\sigma) 2 \beta_{0} r^{2+\sigma} \\
-\left(\theta-2 z+2+\gamma_{1}\right) V_{1} r^{\theta+\gamma_{1}}-\left(\theta-2 z+2+\gamma_{2}\right) V_{2} r^{\theta+\gamma_{2}}=0 .
\end{array}
$$

To obtain an asymptotic HV-AdS solution to above two equations, one finds that the parameters should satisfy the following relations

$$
\begin{aligned}
& \lambda=\theta-2 z+2, \quad \sigma=-2, \quad \gamma_{1}=-\theta, \quad \gamma_{2}=-\theta-2 z+6, \\
& \beta_{0}=(z-1)(\theta-z-2) \quad V_{1}=(\theta-2 z)(\theta-z-2) \quad V_{2}=\frac{(\theta-2 z+2) B^{2} Z_{0}}{-4 z+8} \text {. }
\end{aligned}
$$

Then, we have

$$
f(r)=1-M r^{-\theta+z+2}+\frac{Q^{2} r^{-2 \theta+2 z+2}}{2 Z_{0}(\theta-2)(\theta-z)}+\frac{B^{2} Z_{0} r^{-2 z+6}}{4(2-z)(\theta-3 z+4)} .
$$

In addition, the solution of electric potential $a(r)$ can be obtained from eq. (2.7), given by

$$
a(r)=\mu-\frac{Q}{Z_{0}(z-\theta)} r^{z-\theta} .
$$

So far, we have obtained an analytical dyonic HV-AdS black hole solution. In order to support an analytical dyonic HV-AdS black hole solution from the action (eq. (A.1)), the parameters $\left(\lambda, \sigma, \gamma_{1}, \gamma_{2}, \beta_{0}, V_{1}, V_{2}\right)$ in eq. (3.8) must be expressed in terms of the parameters $z, \theta$ and $B$ in the ansatz (eqs. (A.2) and (A.3)), i.e., satisfying eqs. (A.23). This method has been applied to obtain the analytical Lifshitz-AdS and HV-AdS in [57, 63], respectively.

Open Access. This article is distributed under the terms of the Creative Commons Attribution License (CC-BY 4.0), which permits any use, distribution and reproduction in any medium, provided the original author(s) and source are credited. 


\section{References}

[1] N.E. Hussey, Phenomenology of the normal state in-plane transport properties of high- $T_{c}$ cuprates, J. Phys, Cond. Mat. 20 (2008) 123201 [arXiv:0804.2984].

[2] R A. Cooper et al., Anomalous criticality in the electrical resistivity of $\mathrm{La}_{2-x} \mathrm{Sr}_{x} \mathrm{CuO}_{4}$, Science 323 (2009) 603.

[3] Y. Nakayima et al., Normal-state Hall angle and magnetoresistance in quasi-2D heavy fermion CeCoIn 5 near a quantum critical point, J. Phys. Soc. Jpn. 73 (2004) 5 [cond-mat/0305203].

[4] T.R. Chien, Z.Z. Wang and N.P. Ong, Effects of Zn Impurities on the normal-state hall angle in single-crystal $\mathrm{YBa}_{2} \mathrm{Cu}_{3-x} \mathrm{Zn}_{x} \mathrm{O}_{7-\delta}$, Phys. Rev. Lett. 67 (1991) 2088.

[5] P. W. Anderson, Hall effect in the two-dimensional Luttinger liquid, Phys. Rev. Lett. 67 (1991) 2092.

[6] P. Coleman, A. J. Schofield and A.M. Tsvelik, How should we interpret the two transport relaxation times in the cuprates? J. Phys. Cond. Mat. 8 (1996) 9985 [cond-mat/9609009].

[7] S.A. Hartnoll, Lectures on holographic methods for condensed matter physics, Class. Quant. Grav. 26 (2009) 224002 [arXiv:0903.3246] [INSPIRE].

[8] C.P. Herzog, Lectures on holographic superfluidity and superconductivity, J. Phys. A 42 (2009) 343001 [arXiv:0904.1975] [INSPIRE].

[9] S.A. Hartnoll, J. Polchinski, E. Silverstein and D. Tong, Towards strange metallic holography, JHEP 04 (2010) 120 [arXiv:0912.1061] [INSPIRE].

[10] R.A. Davison, K. Schalm and J. Zaanen, Holographic duality and the resistivity of strange metals, Phys. Rev. B 89 (2014) 245116 [arXiv:1311.2451] [INSPIRE].

[11] S.S. Gubser and F.D. Rocha, Peculiar properties of a charged dilatonic black hole in AdS $S_{5}$, Phys. Rev. D 81 (2010) 046001 [arXiv:0911.2898] [InSPIRE].

[12] S.S. Pal, Model building in AdS/CMT: DC conductivity and Hall angle, Phys. Rev. D 84 (2011) 126009 [arXiv:1011.3117] [INSPIRE].

[13] S.S. Pal, Approximate strange metallic behavior in AdS, arXiv:1202.3555 [INSPIRE].

[14] B. Gouteraux, B.S. Kim and R. Meyer, Charged dilatonic black holes and their transport properties, Fortsch. Phys. 59 (2011) 723 [arXiv:1102.4440] [InSPIRE].

[15] B.S. Kim, E. Kiritsis and C. Panagopoulos, Holographic quantum criticality and strange metal transport, New J. Phys. 14 (2012) 043045 [arXiv: 1012.3464] [INSPIRE].

[16] C. Hoyos, B.S. Kim and Y. Oz, Lifshitz hydrodynamics, JHEP 11 (2013) 145 [arXiv: 1304.7481] [INSPIRE].

[17] B. Goutéraux, Universal scaling properties of extremal cohesive holographic phases, JHEP 01 (2014) 080 [arXiv:1308.2084] [INSPIRE].

[18] B. Goutéraux, Charge transport in holography with momentum dissipation, JHEP 04 (2014) 181 [arXiv: 1401.5436] [INSPIRE].

[19] B.-H. Lee, D.-W. Pang and C. Park, A holographic model of strange metals, Int. J. Mod. Phys. A 26 (2011) 2279 [arXiv:1107.5822] [InSPIRE].

[20] A. Lucas and S. Sachdev, Memory matrix theory of magnetotransport in strange metals, Phys. Rev. B 91 (2015) 195122 [arXiv: 1502.04704] [INSPIRE]. 
[21] A. Karch, Conductivities for hyperscaling violating geometries, JHEP 06 (2014) 140 [arXiv: 1405.2926] [INSPIRE].

[22] S.A. Hartnoll and A. Karch, Scaling theory of the cuprate strange metals, Phys. Rev. B 91 (2015) 155126 [arXiv:1501.03165] [INSPIRE].

[23] M. Blake and A. Donos, Quantum critical transport and the Hall angle, Phys. Rev. Lett. 114 (2015) 021601 [arXiv:1406.1659] [INSPIRE].

[24] D. Vegh, Holography without translational symmetry, arXiv:1301.0537 [INSPIRE].

[25] M. Blake and D. Tong, Universal resistivity from holographic massive gravity, Phys. Rev. D 88 (2013) 106004 [arXiv:1308.4970] [InSPIRE].

[26] A. Amoretti, A. Braggio, N. Maggiore, N. Magnoli and D. Musso, Thermo-electric transport in gauge/gravity models with momentum dissipation, JHEP 09 (2014) 160 [arXiv: 1406.4134] [INSPIRE].

[27] A. Amoretti, A. Braggio, N. Maggiore, N. Magnoli and D. Musso, Analytic dc thermoelectric conductivities in holography with massive gravitons, Phys. Rev. D 91 (2015) 025002 [arXiv: 1407.0306] [INSPIRE].

[28] R.A. Davison, Momentum relaxation in holographic massive gravity, Phys. Rev. D 88 (2013) 086003 [arXiv:1306.5792] [InSPIRE].

[29] H.B. Zeng and J.-P. Wu, Holographic superconductors from the massive gravity, Phys. Rev. D 90 (2014) 046001 [arXiv: 1404.5321] [InSPIRE].

[30] A. Lucas, S. Sachdev and K. Schalm, Scale-invariant hyperscaling-violating holographic theories and the resistivity of strange metals with random-field disorder, Phys. Rev. D 89 (2014) 066018 [arXiv: 1401.7993] [inSPIRE].

[31] M. Baggioli and O. Pujolàs, Electron-phonon interactions, metal-insulator transitions and holographic massive gravity, Phys. Rev. Lett. 114 (2015) 251602 [arXiv:1411.1003] [INSPIRE].

[32] G.T. Horowitz, J.E. Santos and D. Tong, Optical conductivity with holographic lattices, JHEP 07 (2012) 168 [arXiv:1204.0519] [InSPIRE].

[33] G.T. Horowitz, J.E. Santos and D. Tong, Further evidence for lattice-induced scaling, JHEP 11 (2012) 102 [arXiv:1209.1098] [INSPIRE].

[34] G.T. Horowitz and J.E. Santos, General relativity and the cuprates, JHEP 06 (2013) 087 [arXiv: 1302.6586] [INSPIRE].

[35] Y. Ling, C. Niu, J.-P. Wu and Z.-Y. Xian, Holographic lattice in Einstein-Maxwell-Dilaton gravity, JHEP 11 (2013) 006 [arXiv:1309.4580] [INSPIRE].

[36] Y. Ling, C. Niu, J. Wu, Z. Xian and H.-b. Zhang, Metal-insulator transition by holographic charge density waves, Phys. Rev. Lett. 113 (2014) 091602 [arXiv:1404.0777] [INSPIRE].

[37] T. Andrade and B. Withers, A simple holographic model of momentum relaxation, JHEP 05 (2014) 101 [arXiv:1311.5157] [INSPIRE].

[38] A. Donos and J.P. Gauntlett, Holographic Q-lattices, JHEP 04 (2014) 040 [arXiv: 1311.3292] [INSPIRE].

[39] A. Donos and J.P. Gauntlett, Novel metals and insulators from holography, JHEP 06 (2014) 007 [arXiv:1401.5077] [INSPIRE]. 
[40] A. Donos, B. Goutéraux and E. Kiritsis, Holographic metals and insulators with helical symmetry, JHEP 09 (2014) 038 [arXiv: 1406.6351] [INSPIRE].

[41] A. Donos and J.P. Gauntlett, The thermoelectric properties of inhomogeneous holographic lattices, JHEP 01 (2015) 035 [arXiv:1409.6875] [INSPIRE].

[42] X.-H. Ge, Y. Ling, C. Niu and S.-J. Sin, Holographic transports and stability in anisotropic linear axion model, arXiv:1412.8346 [INSPIRE].

[43] L. Cheng, X.-H. Ge and Z.-Y. Sun, Thermoelectric DC conductivities with momentum dissipation from higher derivative gravity, JHEP 04 (2015) 135 [arXiv:1411.5452] [INSPIRE].

[44] M. Blake, D. Tong and D. Vegh, Holographic lattices give the graviton an effective mass, Phys. Rev. Lett. 112 (2014) 071602 [arXiv:1310.3832] [INSPIRE].

[45] A. Amoretti and D. Musso, Universal formulae for thermoelectric transport with magnetic field and disorder, arXiv:1502.02631 [INSPIRE].

[46] M. Blake, A. Donos and N. Lohitsiri, Magnetothermoelectric response from holography, arXiv:1502.03789 [INSPIRE].

[47] K.-Y. Kim, K.K. Kim, Y. Seo and S.-J. Sin, Thermoelectric conductivities at finite magnetic field and the Nernst effect, JHEP 07 (2015) 027 [arXiv: 1502.05386] [INSPIRE].

[48] S.A. Hartnoll, P.K. Kovtun, M. Muller and S. Sachdev, Theory of the Nernst effect near quantum phase transitions in condensed matter and in dyonic black holes, Phys. Rev. B 76 (2007) 144502 [arXiv:0706.3215] [INSPIRE].

[49] S.A. Hartnoll and D.M. Hofman, Locally critical resistivities from Umklapp scattering, Phys. Rev. Lett. 108 (2012) 241601 [arXiv:1201.3917] [INSPIRE].

[50] R.A. Davison and B. Goutéraux, Momentum dissipation and effective theories of coherent and incoherent transport, JHEP 01 (2015) 039 [arXiv:1411.1062] [INSPIRE].

[51] A. Karch and A. O'Bannon, Metallic AdS/CFT, JHEP 09 (2007) 024 [arXiv:0705.3870] [INSPIRE].

[52] A. Donos and J.P. Gauntlett, Thermoelectric DC conductivities from black hole horizons, JHEP 11 (2014) 081 [arXiv:1406.4742] [INSPIRE].

[53] C. Charmousis, B. Gouteraux, B.S. Kim, E. Kiritsis and R. Meyer, Effective holographic theories for low-temperature condensed matter systems, JHEP 11 (2010) 151 [arXiv: 1005.4690] [INSPIRE].

[54] B. Gouteraux and E. Kiritsis, Generalized holographic quantum criticality at finite density, JHEP 12 (2011) 036 [arXiv:1107.2116] [INSPIRE].

[55] B. Gouteraux and E. Kiritsis, Quantum critical lines in holographic phases with (un)broken symmetry, JHEP 04 (2013) 053 [arXiv:1212.2625] [INSPIRE].

[56] T. Ando et al., Mobility of the doped holes and the antiferromagnetic correlations in underdoped High-T $T_{c}$ cuprates, Phys. Rev. Lett. 87 (2001) 017001 [cond-mat/0104163].

[57] M. Alishahiha, E. O Colgain and H. Yavartanoo, Charged black branes with hyperscaling violating factor, JHEP 11 (2012) 137 [arXiv:1209.3946] [INSPIRE].

[58] A. Salvio, Holographic superfluids and superconductors in dilaton-gravity, JHEP 09 (2012) 134 [arXiv:1207.3800] [INSPIRE]. 
[59] A. Salvio, Transitions in dilaton holography with global or local symmetries, JHEP 03 (2013) 136 [arXiv: 1302.4898] [INSPIRE].

[60] D. Roychowdhury, Holographic charge diffusion in non relativistic branes, Phys. Lett. B 744 (2015) 109 [arXiv: 1412.0911] [InSPIRE].

[61] D. Roychowdhury, Hydrodynamics from scalar black branes, JHEP 04 (2015) 162 [arXiv: 1502.04345] [INSPIRE].

[62] E. Mefford and G.T. Horowitz, Simple holographic insulator, Phys. Rev. D 90 (2014) 084042 [arXiv: 1406.4188] [INSPIRE].

[63] J. Tarrio and S. Vandoren, Black holes and black branes in Lifshitz spacetimes, JHEP 09 (2011) 017 [arXiv: 1105.6335] [INSPIRE]. 